PAPERS

\title{
Modelling, analysis and control design of hybrid dynamical systems
}

\author{
Dominik Vošček, Anna Jadlovská, Dominik Grigl'ák ${ }^{*}$
}

\begin{abstract}
This paper introduces a methodology for one of the challenges regarding cyber-physical systems, ie modelling and control design them as hybrid systems. The proposed methodology comprises modules with specific steps to accomplish the tasks. Specifically, the paper aims to utilize hybrid systems framework onto the chosen hydraulic hybrid system with complex dynamics to showcase different aspects of hybrid systems. The mathematical model was derived using hybrid automata framework and then transformed into the linear form either using Jacobi matrices or using linear approximations without Jacobi matrices. After that the system was validated and analysed and the control design utilizing piecewise linear-quadratic regulator optimal control was proposed. Furthermore, parameters of control algorithm were tuned using particle swarm optimization algorithm. The whole logic, system dynamics and constrains are implemented within MATLAB/Simulink simulation environment using $s$-functions. The proposed methodology can be implemented on the various types of cyberphysical systems as far as they can be described as hybrid systems.
\end{abstract}

K e y w ords: cyber-physical system, hydraulic hybrid system, methodology, particle swarm optimization, piecewise affine system, piecewise LQR optimal control

\section{Introduction}

Cyber-physical systems (CPS), defined as an integration of physical processes with computation platforms, are an integral part of the phenomenon Industry 4.0. One of their complexity challenges stated in $[1,2]$ can be formulated as to model and control CPS within hybrid systems framework [3].

This paper focuses specifically on this challenge of CPS from modelling up to the control design as hybrid systems (HS) $[4,5]$. The most convenient hybrid systems framework for such a task is hybrid automata (HA) which naturally deals with a continuous and discrete dynamics of hybrid systems [6]. However, such a mathematical representation is not appropriate for analysis and control design of CPS. For these tasks, different but equivalent mathematical representations were introduced, eg piecewise affine (PWA) systems [7].

There have been proposed several approaches to unify the procedure to model, analyse and design control algorithms for hybrid systems. Within mentioned procedures belong one published in [8], however, this approach does not utilize analysis of the system in the open loop and during control synthesis no metaheuristic algorithms to tune control parameters were used. Another approach, published in [9], utilizes supervisory control of hybrid systems but omits the analysis part. Methodology utilizing modelling and diagnosis of hybrid systems was proposed in $[10]$.

Goal of this article is to propose and introduce the unified methodology for the whole process from modelling up to the control design and therefore cover all the steps to ensure proper design and analysis of CPS as a hybrid system. This process was partially introduced in $[2,11]$ and will be completed with regards to other research challenges. The methodology consists of several steps, namely determination of HA elements such as possible discrete modes and transitions between them, followed by assigning continuous dynamics to these modes. At this point it is possible to simulate and analyse the hybrid system.

After validation of the system, design control utilizing appropriate control algorithms can be implemented onto the hybrid system. Between the most used control algorithms for hybrid systems belong model predictive control based on multiparametric optimization [12] and piecewise optimal linear quadratic (LQR) optimal control [13]. These methods were chosen as representatives of control algorithms for hybrid systems. However, there are many others such as semi-Markov mode switching for linear parameter-varying systems [14] or supervisory control [15].

While designing control law, a metaheuristic method for tuning control law parameters can be utilized. Between these metaheuristic methods belong eg particle swarm optimization (PSO) [16], artificial bee colony [17], ant colony optimization [18] or grey wolf optimization approach [19]. We have chosen PSO algorithm as a representative example for tuning control algorithm parameters. This algorithm was then applied onto all controllable discrete modes of the HS.

*Department of Cybernetics and Artificial Intelligence, Faculty of Electrical Engineering and Informatics, Technical University of Košice, Slovakia, dominik.voscek@tuke.sk, anna.jadlovska@tuke.sk, dominik.griglak@student.tuke.sk 


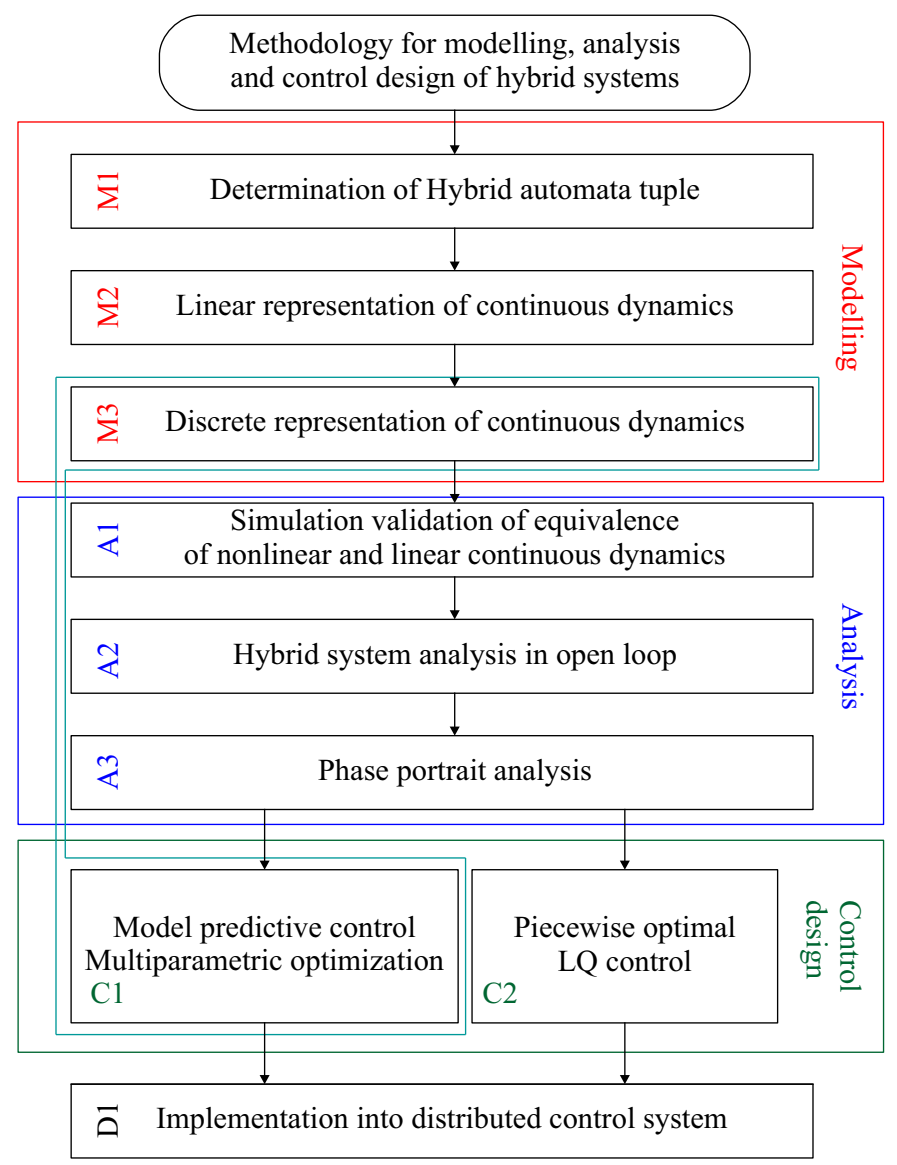

Fig. 1. Methodology for modelling, analysis and control design of hybrid systems

\section{Modelling, analysis and control design of hybrid systems methodology}

The proposed methodology comes out from partially introduced methodology in $[2,11]$ and is comprised of several steps which are grouped into the thematical modules [20], ie Modelling, Analysis and Control Design as seen in Fig. [1]. Module Modelling deals with a derivation of a HS mathematical model in the suitable representation for further analysis and control design. The first step incorporates the definition of a hybrid automaton for CPS (step M1) and all the elements of its tuple.

Hybrid automaton represents an extension of the finite state machines formalism for continuous dynamics in the individual discrete states [21] and is defined as a tuple $H=(Q, X, f$, Init, Dom, E, G, R) [22], where:

- $Q$ represents the finite set of discrete modes $\left\{q_{1}, q_{2}, \ldots, q_{\max }\right\}$

- $X \subseteq \Re^{n}$ denotes state space for continuous dynamics of $H$,

- $\boldsymbol{f}: Q \times \Re^{n} \rightarrow \Re^{n}$ is the collection of vector fields describing the continuous dynamics of the state space vector $\mathbf{x}(t)$ in discrete mode $q$,

- Init $\subseteq Q \times X$ is a set of possible initial hybrid states,
- Dom: $Q \rightarrow 2^{X}$ defines $\forall q \in Q$ a domain in the state space $\operatorname{Dom}(q) \subseteq X$,

- $E \subseteq Q \times Q$ is a set of edges, ie the pairs of discrete modes $\left(q_{i}, q_{j}\right)$ between which a transition is possible,

- $G\left(q_{i}, q_{j}\right)$ represents a guard set, assigning a set of required continuous states for the transition for each edge $\left(q_{i}, q_{j}\right) \in E$,

- $R: E \times \Re^{n} \rightarrow \Re^{n}$ denotes a reset map defining for each edge $\left(q_{i}, q_{j}\right) \in E$ and continuous state $\mathbf{x}(t) \in \Re^{n}$ a change of continuous state between transition from mode $q_{i}$ to mode $q_{j}$.

The state of hybrid automaton $H$ can be then defined as a pair $(q, \mathbf{x}(t)) \in Q \times \Re^{n}$, where $\mathbf{x}(t) \in X$ and $q \in$ $Q$ [23]. It is often suitable and desired to represent the HA in a graphical form such as an oriented graph. Individual members of a set of discrete modes $Q$ are represented as graph nodes and individual possible transitions between these discrete modes are denoted as edges. Each node is determined by an initial set, continuous dynamics and domain. An edge is clearly determined with a member from a guard set and a reset map [22]. An example of such a graphical representation is shown in Fig. 2.

After defining HA tuple of CPS, steps for transforming such a representation into a suitable form such as PWA or discrete PWA system is performed (steps $M 2$ and $M 3$ ). 


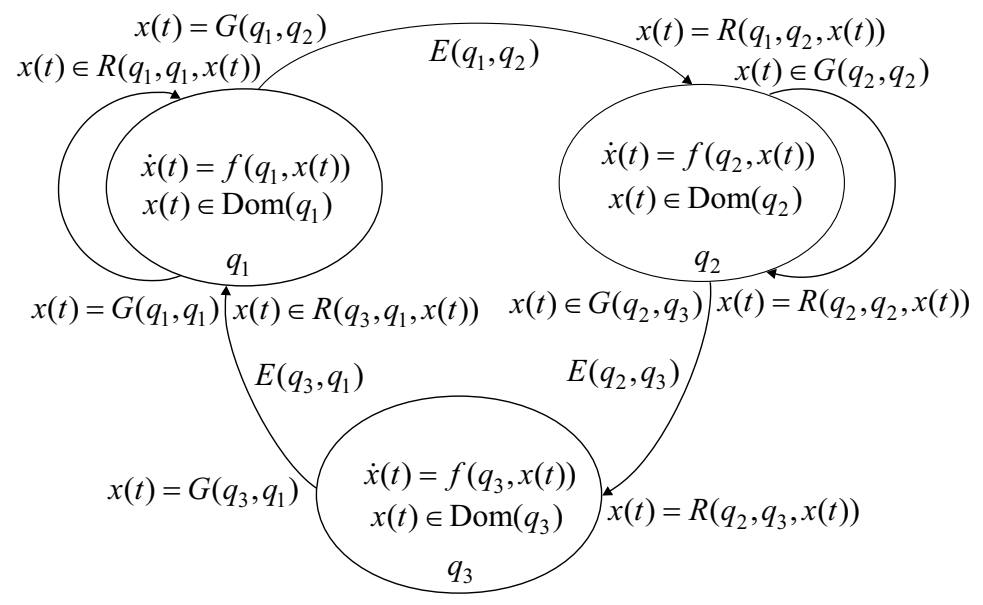

Fig. 2. Graphical representation of hybrid system

Upon obtaining a suitable mathematical representation of CPS as HS it is required to validate the HS. First step within Analysis module is to implement HA into a suitable simulation environment such as MATLAB/Simulink, HYSDEL or others (step A1) [24,25]. Validation can have different forms such as phase portrait analysis or analysis of a time behaviour in the openloop configuration (steps $A 2$ and $A 3$ ). If the mathematical representation does not meet required criteria or does not respect system constraints then steps $M 1-M 3$ have to be repeated.

After validation and analysis of HS, control design can be performed. There are various methods for control design of HS $[12,13]$. Focus of this methodology is however on two control algorithms, ie piecewise optimal linear quadratic control (step $C 2$ ) and model predictive control based on multiparametric optimization (step C1). Both control algorithms come out from PWA or discrete PWA mathematical representation which were obtained in steps $M 2$ and $M 3$ of the proposed methodology. Having designed control algorithms for HS, it is possible to verify them against original nonlinear HS mathematical representation.

Upon completion of all these steps, HS mathematical model, as well as control design algorithms can be implemented within distributed control system of the CPS real application (step $D 1$ ).

\section{Case study for proposed methodology - hydraulic hybrid system}

The proposed methodology is going to be implemented and verified on testbed hydraulic hybrid system (hydraulic HS) except the steps M3 - Discrete representation of continuous dynamics and C1 - Model control based on multiparametric optimization as these two steps are interconnected as seen in Fig. 1. Hydraulic HS represent hybrid system with logical input as well as the system with various discrete modes within which the hydraulic
HS is stable. For some of the discrete modes a linear representation using Jacobians cannot be performed. Therefore a work-around for linearization of those discrete modes is also proposed. Based on these assumptions the overall methodology can be presented.

\subsection{Hydraulic hybrid system as hybrid automata}

HS of interest is represented by hydraulic HS which is composed of two tanks which are interconnected with two valves as seen in Fig. 3. Within this section step $M 1$ - Determination of HA tuple is going to be applied onto the chosen HS. Levels of the liquid in individual tanks are denoted as $h_{1}(t)$ for the first and $h_{2}(t)$ for the second tank. The inflow $q_{0}(t)$ enters the first tank. The tank valves are characterized by the parameters $k_{1}, k_{2}, k_{3}$ with corresponding liquid flow $q_{1}(t), q_{2}(t), q_{3}(t)$. The lower common valve $k_{1}$ can be closed, $i e$ it can be either in open or closed state. Changing its state from open to closed or vice versa is considered to happen in zero time. Valve $k_{2}$ is situated in the height $h_{v}$.

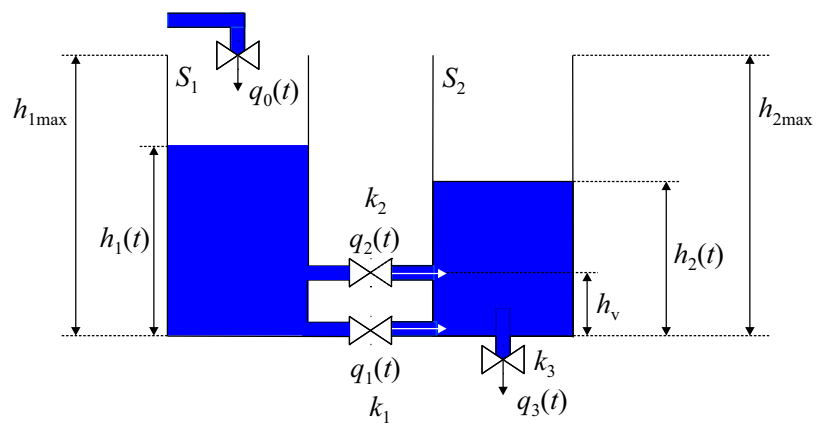

Fig. 3. Scheme of hydraulic hybrid system

Tanks are of cylindrical shape, therefore the crosssection area of the tanks is constant and labeled as $S_{1}$ for the first and $S_{2}$ for the second tank. The maximum heights of the tanks are represented with $h_{1 \max }$ and $h_{2 \max }$ values. 
Table 1. Hydraulic HS physical quantities

\begin{tabular}{lcc} 
Nomen & Range \\
\hline & height of the liquid: & \\
$h_{1}(t)$ & $\langle 0 ; 2\rangle(\mathrm{m})$ & in the first tank \\
$h_{2}(t)$ & $\langle 0 ; 2.4\rangle(\mathrm{m})$ & in the second tank \\
\hline \multicolumn{3}{c}{ inflow to the first tank: } \\
$q_{0}(t)$ & $\langle 0 ; 0.02\rangle\left(\mathrm{m}^{3} / \mathrm{s}\right)$ & through valve \\
\hline & flow: & $k_{1}$ \\
$q_{1}(t)$ & $\langle 0 ; 0.02\rangle\left(\mathrm{m}^{3} / \mathrm{s}\right)$ & $k_{2}$ \\
$q_{2}(t)$ & $\langle 0 ; 0.02\rangle\left(\mathrm{m}^{3} / \mathrm{s}\right)$ & $k_{3}$ \\
$q_{3}(t)$ & $\langle 0 ; 0.02\rangle\left(\mathrm{m}^{3} / \mathrm{s}\right)$ &
\end{tabular}

Table 2. Hydraulic HS discrete modes

\begin{tabular}{lccc}
$\begin{array}{l}\text { Discrete } \\
\text { mode }\end{array}$ & $\begin{array}{c}\text { Condition } \\
\text { for } h_{1}(t)\end{array}$ & $\begin{array}{c}\text { Condition } \\
\text { for } h_{2}(t)\end{array}$ & Valve $k_{1}$ \\
\hline $\mathcal{A}$ & $h_{1}(t) \leq h_{v}$ & $h_{2}(t) \leq h_{v}$ & open \\
$\mathcal{B}$ & $h_{1}(t)>h_{v}$ & $h_{2}(t) \leq h_{v}$ & open \\
$\mathcal{C}$ & $h_{1}(t) \leq h_{v}$ & $h_{2}(t)>h_{v}$ & open \\
$\mathcal{D}$ & $h_{1}(t)>h_{v}$ & $h_{2}(t)>h_{v}$ & open \\
$\mathcal{E}$ & $h_{1}(t) \leq h_{v}$ & $h_{2}(t) \leq h_{v}$ & closed \\
$\mathcal{F}$ & $h_{1}(t)>h_{v}$ & $h_{2}(t) \leq h_{v}$ & closed \\
$\mathcal{G}$ & $h_{1}(t) \leq h_{v}$ & $h_{2}(t)>h_{v}$ & closed \\
$\mathcal{H}$ & $h_{1}(t)>h_{v}$ & $h_{2}(t)>h_{v}$ & closed \\
\hline
\end{tabular}

Nomenclature of physical quantities used in the given hydraulic HS is in Tab. 1. Range defines the state space $X$ for continuous dynamics $\boldsymbol{f}$ and the set "Init" which represent all possible hydraulic HS initial states.

The chosen hydraulic system has a hybrid character, since the system discrete mode changes when one of the water levels $h_{1}(t), h_{2}(t)$ exceeds the height $h_{v}$ in the common upper valve $k_{2}$. The consequence of this is that the system has 4 different discrete modes with the opened lower valve $k_{1}$. With valve $k_{1}$ closed, there are another 4 different discrete modes. In total, the system can be located in eight different discrete mode marked with letters from $\mathcal{A}$ to $\mathcal{H}$.

Conditions needed to be fulfilled in order to change the hydraulic HS dynamics are stated in Tab. 2. Column "Discrete mode" defines the set of discrete states $Q$, each row represent an element of the guard set $G$ and columns "Condition" for $h_{1}(t)$ and $h_{2}(t)$ represent the set Dom.

Hydraulic HS with the respective heights $h_{1}(t), h_{2}(t)$ and the discrete modes which they belong to is depicted in Fig. 4. The closure of the lower valve $k_{1}$ is indicated with the black color of the entire valve. Discrete modes that can not be reached without a system disturbance or without setting the initial conditions directly to those discrete modes are marked as red. Hydraulic HS discrete modes can be described as:

[Mode $\mathcal{A}$ ] - it is not defined which of the tanks levels $h_{1}(t)$ or $h_{2}(t)$ is higher, ie the liquid can flow either from the first tank to the second or vice versa,

[Mode $\mathcal{B}$ ] - the liquid flows from the first tank into the second tank via valve $k_{2}$, the tanks are in interaction over the bottom valve $k_{1}$,

[Mode $\mathcal{C}$ ] - this mode is the opposite of the mode $\mathcal{B}$, ie liquid flows from the second tank into first one through the upper valve $k_{2}$ and the lower valve $k_{1}$. The height of the level $h_{1}(t)$ in the first tank is constantly rising while the level of $h_{2}(t)$ in the second tank is constantly decreasing. This ultimately causes switching hybrid dynamics from mode $\mathcal{C}$ to another mode,

[Mode $\mathcal{D}$ ] - the tanks are in the interaction with each other through the first valve $k_{1}$ and the second valve $k_{2}$. It is not possible to clearly decide which level is higher, [Mode $\mathcal{E}$ ] - this mode is different from mode $\mathcal{A}$ considering the lower valve $k_{1}$ to be closed. The system behaves like two isolated tanks. This is the only mode in which no liquid flows between tanks,

[Mode $\mathcal{F}$ ] - varies from mode $\mathcal{B}$ in terms of the closed bottom valve $k_{1}$,

[Mode $\mathcal{G}$ ] - differs from mode $\mathcal{C}$ with closed valve $k_{1}$,

[Mode $\mathcal{H}$ ] - varies from mode $\mathcal{D}$ in terms of closed lower valve $k_{1}$. Level in the second tank $h_{2}(t)$ may be higher than the level in the first tank $h_{1}(t)$.

Continuous dynamics $\boldsymbol{f}$ of the each individual discrete state can be introduced as a pair of differential equations

$$
\begin{aligned}
& \dot{h}_{1}(t)=f_{1 n}\left(q_{0}(t), h_{1}(t), h_{2}(t)\right), \\
& \dot{h}_{2}(t)=f_{2 n}\left(q_{0}(t), h_{1}(t), h_{2}(t)\right),
\end{aligned}
$$

where $n \in\{\mathcal{A}, \mathcal{B}, \mathcal{C}, \mathcal{D}, \mathcal{E}, \mathcal{F}, \mathcal{G}, \mathcal{H}\}$. Individual parameters and differential equations are stated in Tab. 3 and Tab. 4.
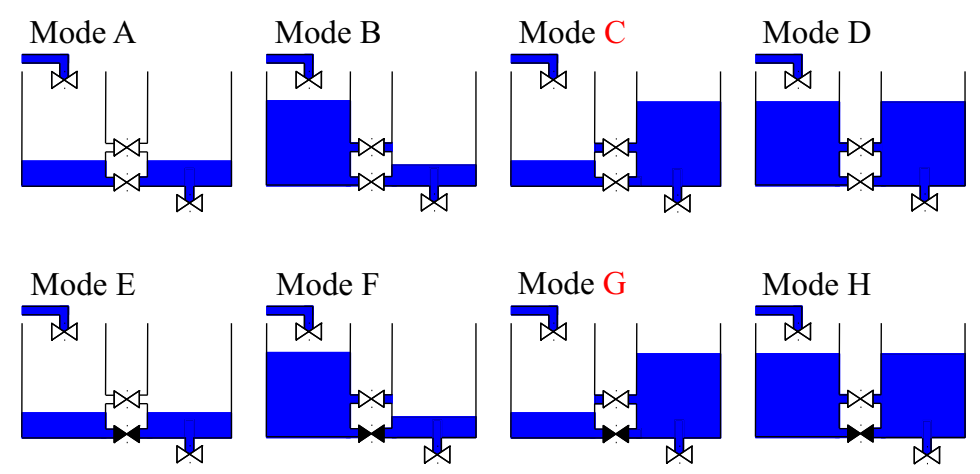

Fig. 4. Graphical depiction of hydraulic HS individual discrete modes 
Table 3. Hydraulic HS physical quantities

maximum height of tanks: $h_{1 \max }, h_{2 \max }=2 \mathrm{~m}, 2.4 \mathrm{~m}$ maximum inflow: $q_{0 \max }=0.02 \mathrm{~m}^{3} / \mathrm{s}$ height of the second valve: $h_{v}=1 \mathrm{~m}$ tank cross sectional area: $S_{1}, S_{2}=1 \mathrm{~m}^{2}$ flow resistance constants: $k_{1}, k_{2}, k_{3}=0.0126 \mathrm{~m}^{2.5} / \mathrm{s}$

Table 4. Hydraulic HS continuous dynamics

\begin{tabular}{|c|c|}
\hline$f_{1 \mathcal{A}}:$ & $\frac{q_{0}}{S_{1}}-\frac{k_{1} \operatorname{sign}\left(h_{1}-h_{2}\right) \sqrt{\left|h_{1}-h_{2}\right|}}{S_{1}}$ \\
\hline$f_{24}:$ & $\underline{k_{1} \operatorname{sign}\left(h_{1}-h_{2}\right) \sqrt{\left|h_{1}-h_{2}\right|}}-\underline{k_{3} \sqrt{h_{2}}}$ \\
\hline$f_{1 \mathcal{B}}:$ & $\frac{q_{0}}{S_{1}}-\frac{k_{1} \sqrt{h_{1}-h_{2}}}{S_{1}}-\frac{k_{2} \sqrt{h_{1}-h_{v}}}{S_{1}}$ \\
\hline$f_{2 \mathcal{B}}:$ & $\frac{k_{1} \sqrt{h_{1}-h_{2}}}{S_{2}}+\frac{k_{2} \sqrt{h_{1}-h_{v}}}{S_{2}}-\frac{k_{3} \sqrt{h_{2}}}{S_{2}}$ \\
\hline$f_{1 \mathcal{C}}:$ & $\frac{q_{0}}{S_{1}}+\frac{k_{1} \sqrt{h_{2}-h_{1}}}{S_{1}}+\frac{k_{2} \sqrt{h_{2}-h_{v}}}{S_{1}}$ \\
\hline$f_{2 \mathcal{C}}:$ & $-\frac{k_{1} \sqrt{h_{2}-h_{1}}}{S_{2}}-\frac{k_{2} \sqrt{h_{2}-h_{v}}}{S_{2}}-\frac{k_{3} \sqrt{h_{2}}}{S_{2}}$ \\
\hline$f_{1 \mathcal{D}}:$ & $\frac{q_{0}}{S_{1}}-\frac{\left(k_{1}+k_{2}\right) \operatorname{sign}\left(h_{1}-h_{2}\right) \sqrt{\left|h_{1}-h_{2}\right|}}{S_{1}}$ \\
\hline$f_{2 \mathcal{D}}:$ & $\frac{\left(k_{1}+k_{2}\right) \operatorname{sign}\left(h_{1}-h_{2}\right) \sqrt{\left|h_{1}-h_{2}\right|}}{S_{2}}-\frac{k_{3} \sqrt{h_{2}}}{S_{2}}$ \\
\hline$f_{1 \mathcal{E}}:$ & $\begin{array}{r}\frac{q_{0}}{S_{1}} \\
-\frac{k_{3} \sqrt{h_{2}}}{S_{2}}\end{array}$ \\
\hline$f_{1 \mathcal{F}}:$ & $\frac{q_{0}}{S_{1}}-\frac{k_{2} \sqrt{h_{1}-h_{v}}}{S_{1}}$ \\
\hline$f_{2 \mathcal{F}}:$ & $\frac{k_{2} \sqrt{h_{1}-h_{v}}}{S_{2}}-\frac{k_{3} \sqrt{h_{2}}}{S_{2}}$ \\
\hline$f_{1 \mathcal{G}}:$ & $\frac{q_{0}}{S_{1}}+\frac{k_{2} \sqrt{h_{2}-h_{v}}}{S_{1}}$ \\
\hline$f_{2 \mathcal{G}}:$ & $-\frac{k_{2} \sqrt{h_{2}-h_{v}}}{S_{2}}-\frac{k_{3} \sqrt{h_{2}}}{S_{2}}$ \\
\hline$f_{1 \mathcal{H}}:$ & $\frac{q_{0}}{S_{1}}-\frac{k_{2} \operatorname{sign}\left(h_{1}-h_{2}\right) \sqrt{\left|h_{1}-h_{2}\right|}}{S_{1}}$ \\
\hline$f_{2 \mathcal{H}}:$ & $\frac{k_{2} \operatorname{sign}\left(h_{1}-h_{2}\right) \sqrt{\left|h_{1}-h_{2}\right|}}{S_{2}}-\frac{k_{3} \sqrt{h_{2}}}{S_{2}}$ \\
\hline
\end{tabular}

Table 5. Hydraulic HS parameters

\begin{tabular}{lccc}
\hline Mode & $h_{1 s n}(\mathrm{~m})$ & $h_{2 s n}(\mathrm{~m})$ & $q_{0 s n}\left(\mathrm{~m}^{3} / \mathrm{s}\right)$ \\
\hline $\mathcal{A}$ & 0.6 & 0.3 & 0.0069 \\
$\mathcal{B}$ & 1.0167 & 0.6 & 0.0098 \\
$\mathcal{D}$ & 1.5 & 1.2 & 0.0139 \\
$\mathcal{F}$ & 1.5 & 0.5 & 0.0089 \\
$\mathcal{H}$ & 2.2 & 1.1 & 0.0133 \\
\hline
\end{tabular}

Regarding the amount of notation, physical quantities $q_{0}(t), h_{1}(t)$ and $h_{2}(t)$ are shortened to $q_{0}, h_{1}$ and $h_{2}$.

Graphical representation of the aforementioned discrete modes is depicted in Fig. 5 with clearly visible pair of discrete modes comprising the set of edges $E$ of $\mathrm{HA}$ tuple.

\subsection{Linear representation of continuous dynamics of hydraulic hybrid system}

Upon completion of nonlinear mathematical model derivation of hydraulic HS, it is needed to obtain a linear approximation as this step is a prerequiste for designing control law. To analyze the system with an input $u(t)$ represented with the inflow $q_{0}(t)$ to the system, it is necessary to determine equilibrium points $h_{1 s n}, h_{2 s n}$ of $h_{1}(t)$ and $h_{2}(t)$ tank levels and steady inflow $q_{0 s n}$ for individual discrete modes. This incorporates step $M 2$ Linear representation of continuous dynamics from proposed methodology.

The hydraulic HS is within equilibrium point if the same volume of liquid $q_{0 s n}$ flows into the first tank as it flows out from the second tank via valve $k_{3}$, ie $q_{3}(t)$. The same principle applies to tank level heights $h_{1}(t), h_{2}(t)$ which must not change, ie their derivatives are equal to zero, $\dot{h}_{1}(t)=0, \dot{h}_{2}(t)=0$, [26].

For all discrete modes with an existing equilibrium point, ie $\mathcal{A}, \mathcal{B}, \mathcal{D}, \mathcal{F}, \mathcal{H}$, the procedure was to determine the steady state value in the second tank $h_{2 s n}$, steady the value in the first tank $h_{1 s n}$ and the steady flow $q_{0 s n}$ were then calculated as it is shown in Tab. 5 .

Based on these values, Jacobi matrices $\boldsymbol{A}_{n}$ and $\boldsymbol{B}_{n}$ were calculated for each discrete mode where $n \in\{\mathcal{A}, \mathcal{B}, \mathcal{D}, \mathcal{F}, \mathcal{H}\}$.

$$
\begin{aligned}
\boldsymbol{A}_{n} & =\left(\begin{array}{ll}
\frac{\partial f_{1 n}}{\partial h_{1}} & \frac{\partial f_{1 n}}{\partial h_{2}} \\
\frac{\partial f_{2 n}}{\partial h_{1}} & \frac{\partial f_{2 n}}{\partial h_{2}}
\end{array}\right)_{q_{0 s n}, h_{1 s n}, h_{2 s n}}, \\
\boldsymbol{B}_{n} & =\left(\begin{array}{l}
\frac{\partial f_{1 n}}{\partial q_{0}} \\
\frac{\partial f_{2 n}}{\partial q_{0}}
\end{array}\right)_{q_{0 s n}, h_{1 s n}, h_{2 s n} .} .
\end{aligned}
$$

After this procedure deviation linear approximation of the nonlinear hydraulic HS can be written as

$$
\Delta \dot{\boldsymbol{h}}_{n}(t)=\boldsymbol{A}_{n} \Delta \boldsymbol{h}_{n}(t)+\boldsymbol{B}_{n} \Delta q_{0 n}(t) .
$$

Discrete modes $\mathcal{C}$ and $\mathcal{G}$ do not have an equilibrium point, and discrete mode $\mathcal{E}$ has an equilibrium point whose Jacobian can not be created for. The square root functions $f_{i}(x)$ were approximated by replacing them with linear functions $f l_{i}(x)$ regarding the following conditions: they share the same domain, ie $D_{i}(f)=$ $D_{i}(f l)=\left\langle x_{1} ; x_{2}\right\rangle$ and they share the same functional values within the boundaries of this domain, ie $f_{i}\left(x_{1}\right)=$ $f l_{i}\left(x_{1}\right) ; f_{i}\left(x_{2}\right)=f l_{i}\left(x_{2}\right),[27]$.

According to Tab. 3 , regarding parameters $h_{1 \max }$ and $h_{2 \max }$ a substitution can be introduced

$$
l_{m}=\frac{\sqrt{h_{m \max }}}{h_{m \max }}, \quad m \in 1,2,
$$




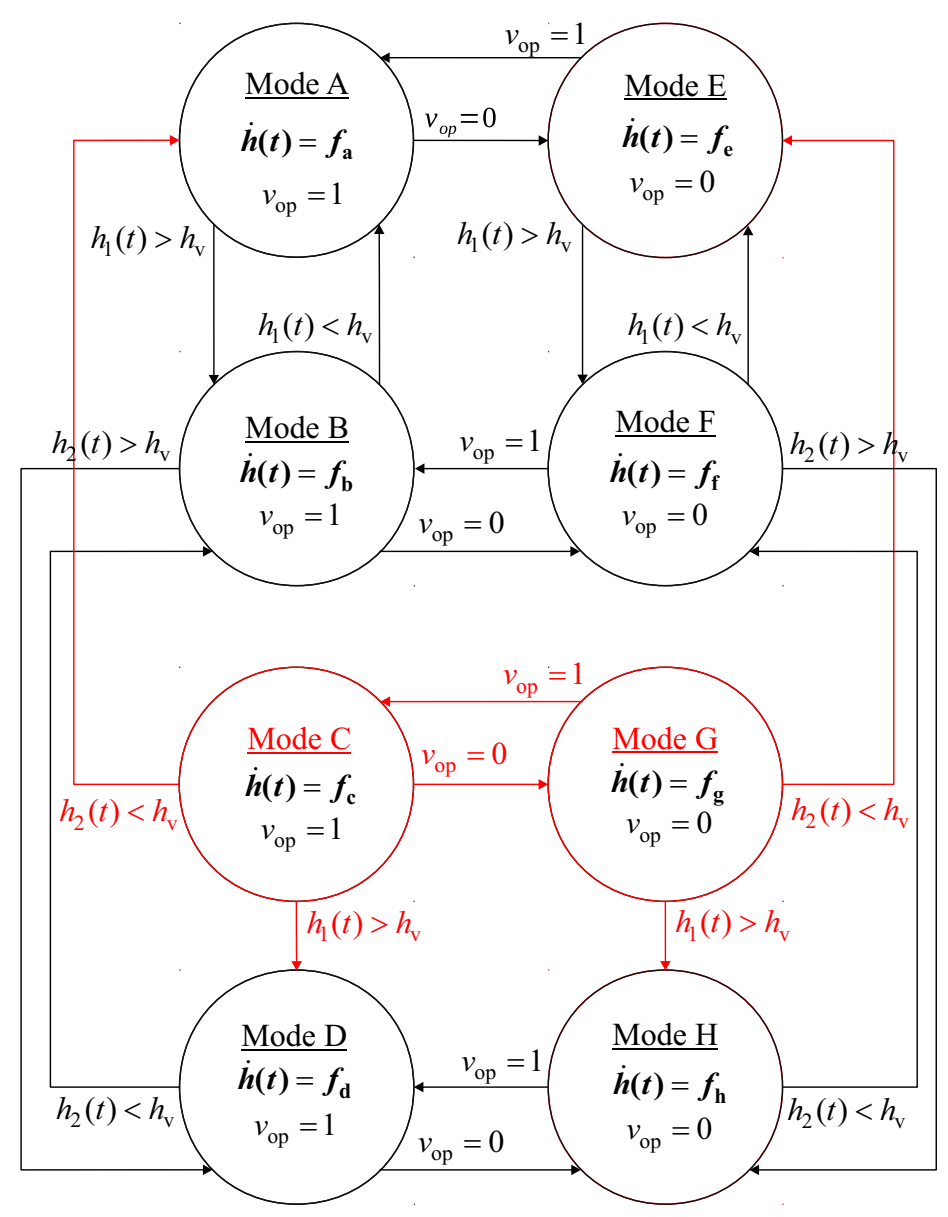

Fig. 5. Transition graph of hydraulic HS

Table 6. Linear approximation of $\mathcal{C}, \mathcal{E}, \mathcal{G}$ discrete modes

\begin{tabular}{ll}
\hline Mode & Linear Approximation \\
\hline $\mathcal{C}$ & $\dot{h}_{1}=\frac{q_{0}}{S_{1}}+\frac{l_{2} k_{1}\left(h_{2}-h_{1}\right)}{S_{1}}+\frac{l_{1} k_{2}\left(h_{2}-h_{v}\right)}{S_{1}}$ \\
& $\dot{h}_{2}=-\frac{l_{2} k_{1}\left(h_{1}-h_{2}\right)}{S_{2}}-\frac{l_{1} k_{2}\left(h_{2}-h_{v}\right)}{S_{2}}-\frac{l_{2} k_{3} h_{2}}{S_{2}}$ \\
$\mathcal{E}$ & $\dot{h}_{1}=\frac{q_{0}}{S_{1}}$ \\
& $\dot{h}_{2}=\frac{-l_{1} k_{3} h_{2}}{S_{2}}$ \\
$\mathcal{G}$ & $\dot{h}_{1}=\frac{q_{0}}{S_{1}}+\frac{l_{1} k_{2}\left(h_{2}-h_{v}\right)}{S_{1}}$ \\
& $\dot{h}_{2}=-\frac{l_{1} k_{2}\left(h_{1}-h_{v}\right)}{S_{2}}-\frac{l_{1} k_{3} h_{2}}{S_{2}}$
\end{tabular}

and based on this substitution a linear approximation for discrete modes without Jacobi matrices, ie $\mathcal{C}, \mathcal{E}, \mathcal{G}$, can be defined as stated in Tab. 6 .

This step closes up module Modelling of CPS as HS within the proposed methodology. Now it is possible to analyse the hydraulic HS, validate it and design suitable control algorithms.
3.3 Validation and phase portrait analysis of hydraulic $H S$

Upon completion of module Modelling, steps within module Analysis can be performed. Inevitable prerequisite for analysis is to implement HS within simulation environment $e g$ toolbox HYSDEL or utilizing $s$-functions. It is then possible to conduct steps from Analysis module. Phase portrait analysis serves to examine state space trajectories of the system. This method is interesting in terms of changing system's dynamics and therefore is included within the proposed methodology.

The hydraulic HS is of the second order, therefore the phase space of this system is a two-dimensional plane and it is possible to create a phase portrait for each discrete mode in one oriented graph [28]. Because the hydraulic HS has a discrete event that is represented with the logical state of the valve $k_{1}$, it is possible to distinguish between the phase portrait for the continuous dynamics of discrete modes $\mathcal{A}, \mathcal{B}, \mathcal{C}, \mathcal{D}$ and the phase portrait for the continuous dynamics of discrete modes $\mathcal{E}, \mathcal{F}, \mathcal{G}, \mathcal{H}$. Phase portraits were obtained for an autonomous hydraulic HS, ie zero inflow $q_{0}(t)=0$ is considered into the first tank. Tank levels $h_{1}(t)$ and $h_{2}(t)$ with open valve $k_{1}$ tend to 

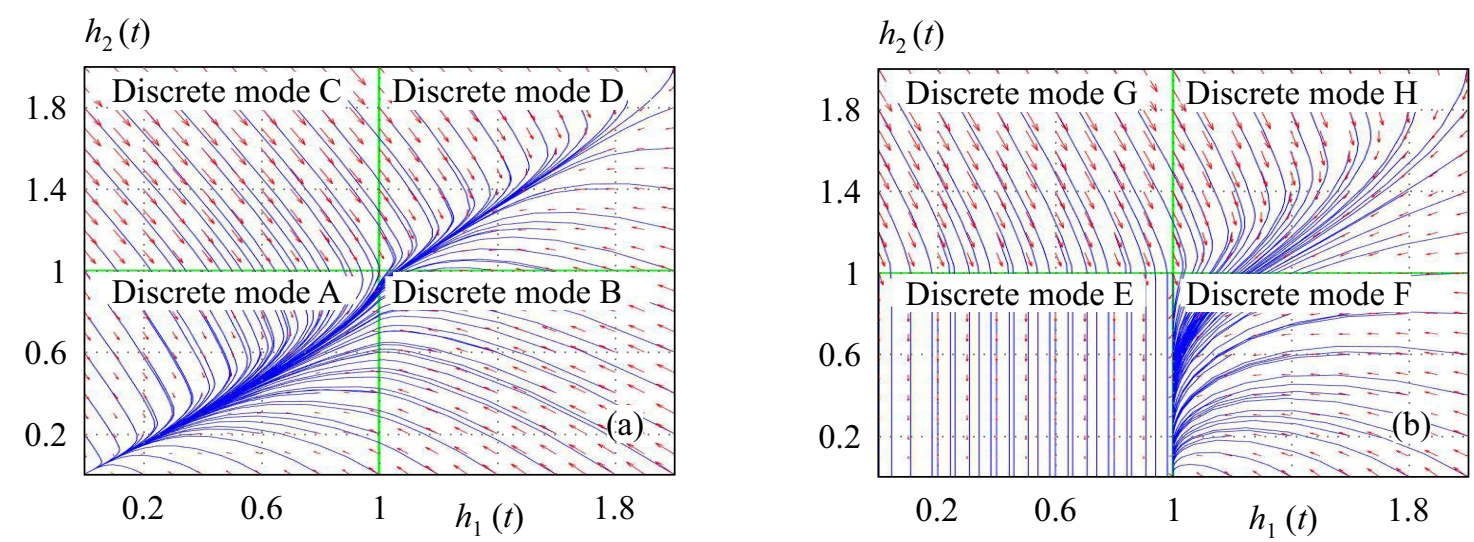

Fig. 6. Hydraulic HS phase portrait for nonlinear representation
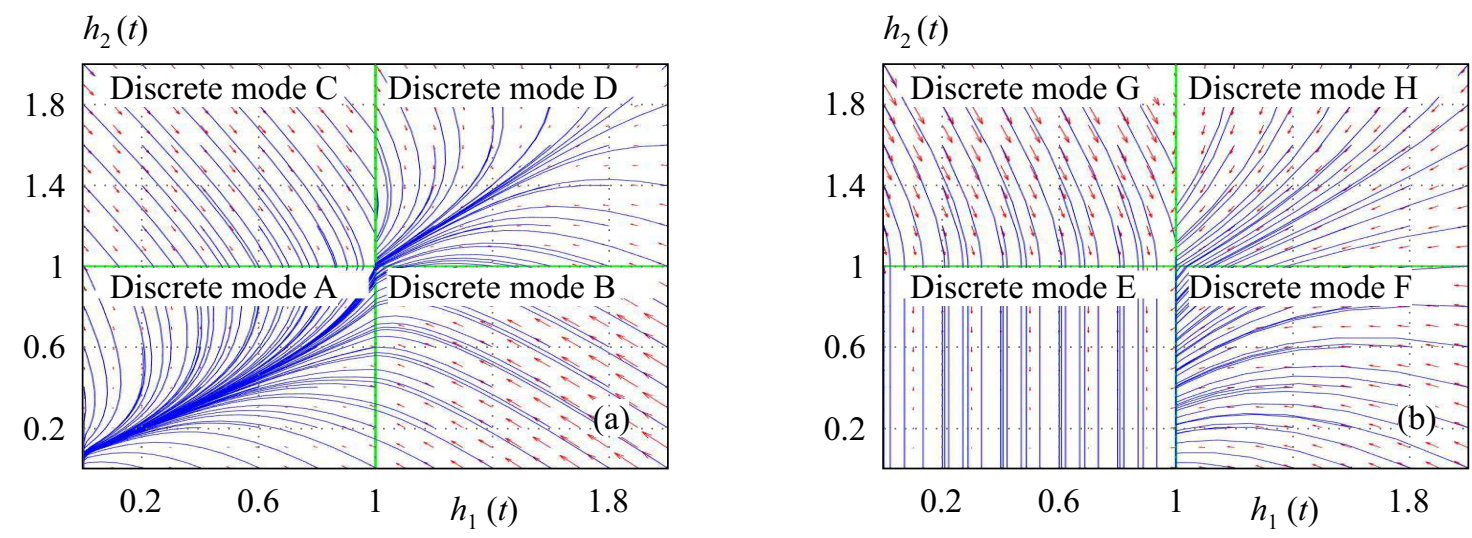

Fig. 7. Hydraulic HS phase portrait for linear representation

flow out of the tanks at the approximately same rate as it can be seen in the left part of Fig. 6 .

With the closed valve $k_{1}$ it is impossible for the first tank to flow out when the condition $h_{1}(t)<h_{v}$ holds which is illustrated for discrete mode $\mathcal{E}$ in the right part of the Fig. 6. In contrast, if for the height of the first tank the condition $h_{1}(t)>h_{v}$ holds, the hydraulic HS stays in the state where the height of the level in the first tank $h_{1}(t)=h_{v}$. This phenomenon can be observed when the hydraulic HS switches from discrete mode $\mathcal{F}$ to mode $\mathcal{E}$, where all phase trajectories point to the point $\left(h_{v} 0\right)^{\top}$. The height of the level in the second tank $h_{2}(t)$ is after some time at zero level since zero inflow $q_{0}(t)=0$ is considered. The individual discrete modes of the hydraulic HS are distinguished from each other with the green lines.

To compare the accuracy of the linear approximation of the hydraulic HS nonlinear model, phase portrait of the linear model was obtained in the same way as the phase portrait of the nonlinear model. The phase portrait of the linear approximation of the hydraulic HS is depicted in Fig. 7.

The inaccuracy of the linear approximation occurs mainly at the point $\left(h_{1}(t) h_{2}(t)\right)^{\top}=\left(\begin{array}{ll}0 & 0\end{array}\right)^{\top}$, when both tanks are empty and the valve $k_{1}$ is opened. Another inaccuracy is apparent during switching between discrete mode $\mathcal{C}$ and mode $\mathcal{D}$. Comparing linear approximation with nonlinear model of hydraulic HS with closed valve $k_{1}$ differences in switching between discrete modes $\mathcal{G}$ and $\mathcal{H}$ is visible continuously switching between these modes. Such a phenomenon is not occurring in the nonlinear model of hydraulic HS phase space, where the system has an effort to switch from discrete mode $\mathcal{G}$ to mode $\mathcal{H}$ and to stay in this discrete mode.

Despite these inaccuracies, it can be concluded that the linear approximation of hydraulic HS is sufficiently precise. Therefore, it is possible to design control algorithms utilizing the linear approximation of nonlinear hydraulic HS and then apply these algorithms onto the nonlinear hydraulic HS. An analysis of the linear approximation by means of the phase plane also verified that the time behaviour in the discrete modes without an existing equilibrium point that were approximated by square root substitution, is comparable to time behaviour of the nonlinear hydraulic HS.

\subsection{Analysis of hydraulic HS in the openloop}

Open-loop response, step A2 - Hybrid system analysis in openloop within the the proposed methodology, serves to follow the transitions between individual discrete modes with the given input. By applying a steady flow $q_{0 s n}$ of the $n$-th discrete mode $n \in\{\mathcal{A}, \mathcal{B}, \mathcal{D}, \mathcal{F}, \mathcal{H}\}$ 

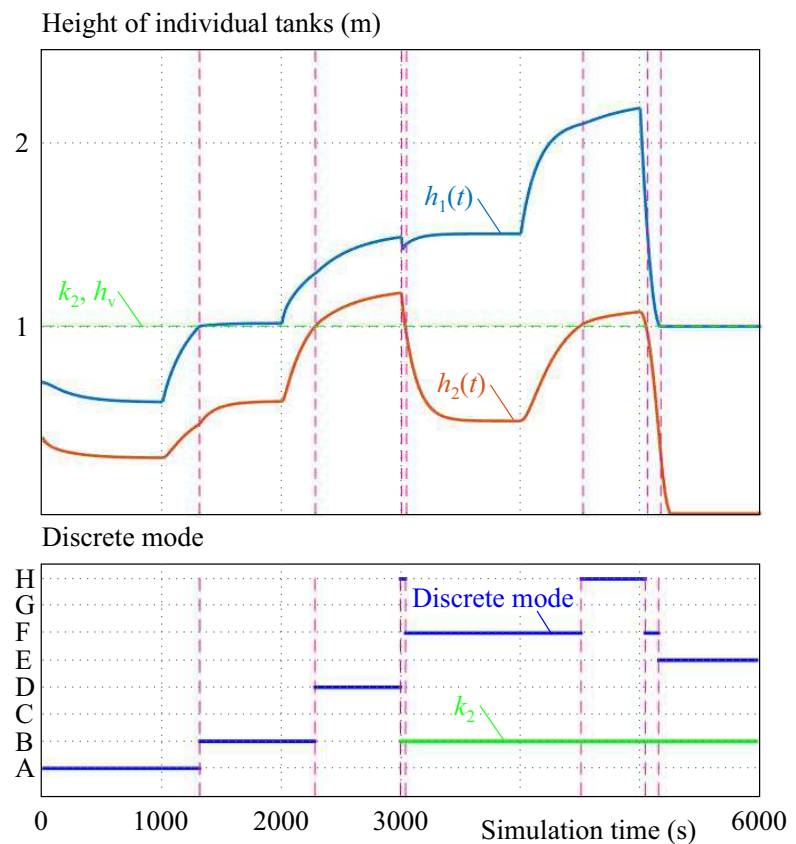

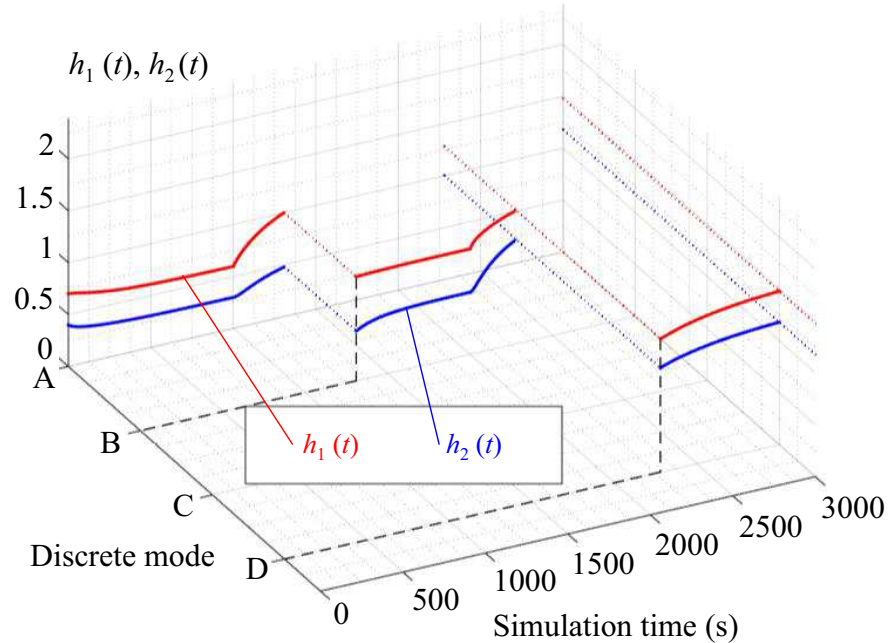

Fig. 9. 3D time evolution of hydraulic HS

Fig. 8. Hydraulic HS phase portrait for linear representation

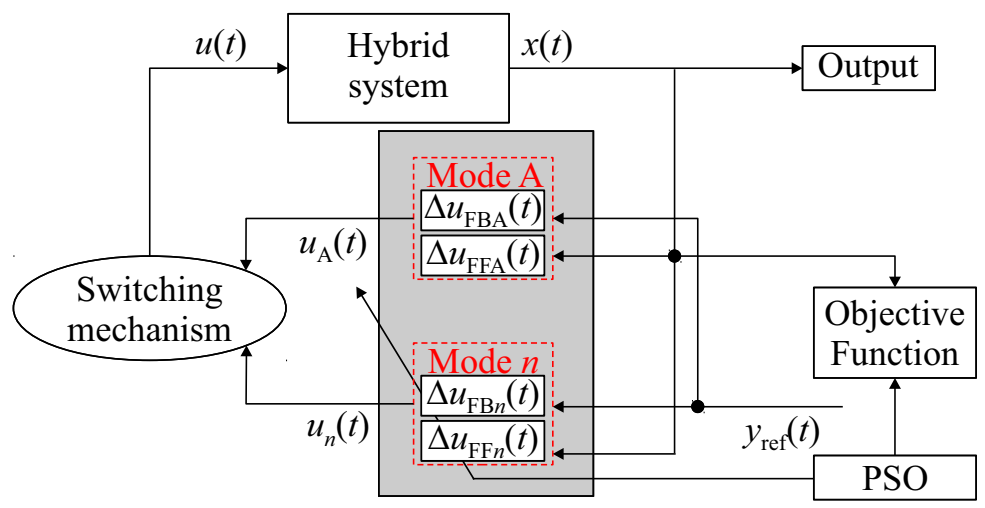

Fig. 10. Piecewise linear-quadratic optimal control schem

onto the hydraulic HS, the system will eventually enter the $n$-th discrete mode. Transitions between hydraulic HS discrete modes is shown in Fig. 8, where upper graph shows tank level heights $h_{1}(t)$ and $h_{2}(t)$. Green dotted line shows the height $h_{v}$ which the valve $k_{2}$ is positioned in. The pink lines represent the time at which a transition between discrete modes happens. Bottom graph shows the discrete modes of the hydraulic HS which the system is located in. Discrete modes are determined according to conditions in Tab. 2. Changing the opening logical state of the valve $k_{1}$ is characterized with the green line.

The transition between discrete modes occurs when the height of a tank level $h_{1}(t)$ or $h_{2}(t)$ exceeds the height $h_{v}$ or after the change of valve $k_{1}$ logical state. The heights of tank levels $h_{1}(t)$ and $h_{2}(t)$ can be also displayed in their respective discrete modes in the $3 \mathrm{D}$ chart, the third dimension being discrete modes of hydraulic HS. Such a graph is displayed in Fig. 9.
After validation and analysis of the hydraulic HS within Analysis module (step $A 1-A 3$ ) in its nonlinear and/or linear form, control algorithms can be designed.

\subsection{Piecewise LQR optimal control}

As far as design control of hybrid systems is concerned, various control algorithms can be utilizied, eg model predictive control based on multiparametric optimization [12] or piecewise linear quadratic optimal control (PWLQR) [29], the latter will be applied onto the hydraulic HS (step $C 2$ within the methodology). Since Jacobi matrices for hydraulic HS could be created only for discrete modes $\{\mathcal{A}, \mathcal{B}, \mathcal{D}, \mathcal{F}, \mathcal{H}\}$, state space control input was designed only for these modes. State space vector for hydraulic HS is defined as $\mathbf{x}(t)=\left(h_{1}(t) h_{2}(t)\right)^{\top}$ and input as $u(t)=q_{0}(t)$.

The main difference against simple LQR control algorithm is in the minimazing objective function within 
each Domain element of the HA tuple. The objective of the PWLQR is then to bring the system to $\Delta \mathbf{x}(\infty)=0$ from an arbitrary initial state $\Delta \mathbf{x}(0)$, while limiting the cost

$$
J(\mathbf{x}, u)(t)=\int_{0}^{\infty}\left(\Delta \mathbf{x}^{\top} \boldsymbol{Q}_{n} \Delta \mathbf{x}+\Delta u^{\top} \boldsymbol{R}_{n} \Delta u\right) \mathrm{d} t,
$$

where $\boldsymbol{Q}_{n}$ and $\boldsymbol{R}_{n}$ are weight matrices for states and input for the $n$-th discrete mode. Subsequently it is possible to calculate the feedback gain $\boldsymbol{k}(t)=\boldsymbol{R}^{-1} \boldsymbol{B}^{\top} \boldsymbol{S}(t)$ where the matrix $\boldsymbol{S}(t)$ is the solution of the Riccati differential equation. Considering unbounded time, Riccati differential equation can be transformed into the algebraic Riccati equation

$$
0=-\boldsymbol{Q}_{n}-\boldsymbol{A}_{n}^{\top} \boldsymbol{S}_{n}-\boldsymbol{S}_{n} \boldsymbol{A}_{n}+\boldsymbol{S}_{n} \boldsymbol{B}_{n} \boldsymbol{R}_{n}^{-1} \boldsymbol{B}_{n}^{\top} \boldsymbol{S}_{n},
$$

where $\boldsymbol{A}_{n}, \boldsymbol{B}_{n}$ are dynamics matrices for individual discrete modes. State feedback control can be then defined as

$$
\Delta u_{F B n}(t)=-\boldsymbol{k}_{n}^{\top} \Delta \mathbf{x}(t) .
$$

If required, any state variable from the state vector $\mathbf{x}(t)$ can be steered to the new required value, ie new steady state $y_{r e f}(t)$. Output of the hydraulic HS is defined as $y(t)=h_{2}(t)$. Based on this, steady state $y_{\text {ref }}(t)=h_{2 r e f}(t)$ can be achieved via the addition of the feed-forward gain $N$ for the $n$-th mode

$$
\boldsymbol{N}_{n}=-\boldsymbol{B}_{n}^{-1}\left(\boldsymbol{A}_{n}-\boldsymbol{B}_{n} \boldsymbol{k}_{n}\right) \boldsymbol{C}_{n}^{-1},
$$

and the control law for feed-forward control input is of the form

$$
\Delta u_{F F n}(t)=N_{n} \Delta y_{\mathrm{ref}}(t) .
$$

The total control input $u(t)$ applied on the system is then the sum of the feedback and feed-forward control inputs

$$
\Delta u_{n}(t)=\Delta u_{F B n}(t)+\Delta u_{F F n}(t) .
$$

The control input defined in (10) holds for linear approximation of the nonlinear dynamical system. However designed control algorithm is applied on the nonlinear form of hydraulic HS. Therefore, state space vector $\mathbf{x}(t)$ and input $u(t)$ within control scheme in Fig. 10 are not considered in their deviation form.

For discrete modes without the equilibrium point, the maximum constant input was applied according to Tab. 5. This input eventually forces the hydraulic HS to transit into the discrete mode with the designed state space control input. The initial conditions $h_{1}(0), h_{2}(0)$ in the simulation were also set so that the hydraulic HS is within the discrete mode with designed control algorithm. This holds for the discrete modes with existing Jacobi matrices.
3.5.1 The Particle Swarm Optimization within the LQR Controller design

Particle Swarm Optimization (PSO) algorithm represents a heuristic search technique used to find the solution for optimization problems. PSO algorithm starts with creating an initial population (swarm) within an initialization region. This population consists of randomly generated particles which search the optimum solution by navigating in the problem space and are defined by position $x_{i}(k)$ and velocity $v_{i}(k)$, where $k$ is the iteration number. The position of each particle represents a candidate for solution of the optimization problem represented by the objective function $J_{P S O}(k)$. The fitness of the each particle is calculated according to the objective function $J_{P S O}(k)$ and the best position $p_{i}(k)$ ever visited by that particle is determined. After the determination of the $p_{i}(k)$ value of each particle in the population, the best position of any particle $g_{i}(k)$ is determined. The positions and the velocities of the particles are calculated as

$$
\begin{aligned}
& v_{i}(k+1)=v_{i}(k)+c_{1} r_{1}\left(p_{i}(k)\right.\left.-x_{i}(k)\right)+ \\
&+c_{2} r_{2}\left(g_{i}(k)-x_{i}(k)\right), \\
& x_{i}(k+1)=x_{i}(k)+v_{i}(k+1), \quad i=1, \ldots, m,
\end{aligned}
$$

where $m$ is the number of particles, $c_{1}$ and $c_{2}$ are the positive constants, $r_{1}$ and $r_{2}$ are two random numbers within the range $[0,1], p_{i}(k)$ is the best position of the $i$-th particle and $g_{i}(k)$ is the best particle among all the particles.

According to optimization problem of tuning parameters of LQR controller with $\boldsymbol{Q}=\left(Q_{11} 0 ; 0 Q_{22}\right)$, particles can be defined as

$$
\mathbf{x}_{i n}(k)=\left[Q_{11 n}(k), Q_{22 n}(k), R_{n}(k)\right]^{\top},
$$

with the objective function stated as

$J_{P S O n}(k)=h_{2 n t_{s}}(k)+h_{2 n t_{r}}(k)+h_{2 n t_{p}}(k)+h_{2 \mathrm{n} \max }(k)$,

where $t_{s}$ is the rise time, $t_{r}$ is the settling time, $t_{p}$ is the peak time, max is the maximum overshoot of individual discrete mode and $n \in\{\mathcal{A}, \mathcal{B}, \mathcal{D}, \mathcal{F}, \mathcal{H}\}$. Numerical values of PSO algorithm are stated in Tab. 7.

Table 7. Hydraulic HS parameters

\begin{tabular}{lc} 
Variable & Range \\
\hline$k_{\max }$ & 3 \\
$c_{1}$ & 2 \\
$c_{2}$ & 2 \\
$Q_{i i, \min }$ & 0.5 \\
$Q_{i i, \max }$ & 1500 \\
$R_{\min }$ & 0.5 \\
$R_{\max }$ & 50 \\
\hline
\end{tabular}




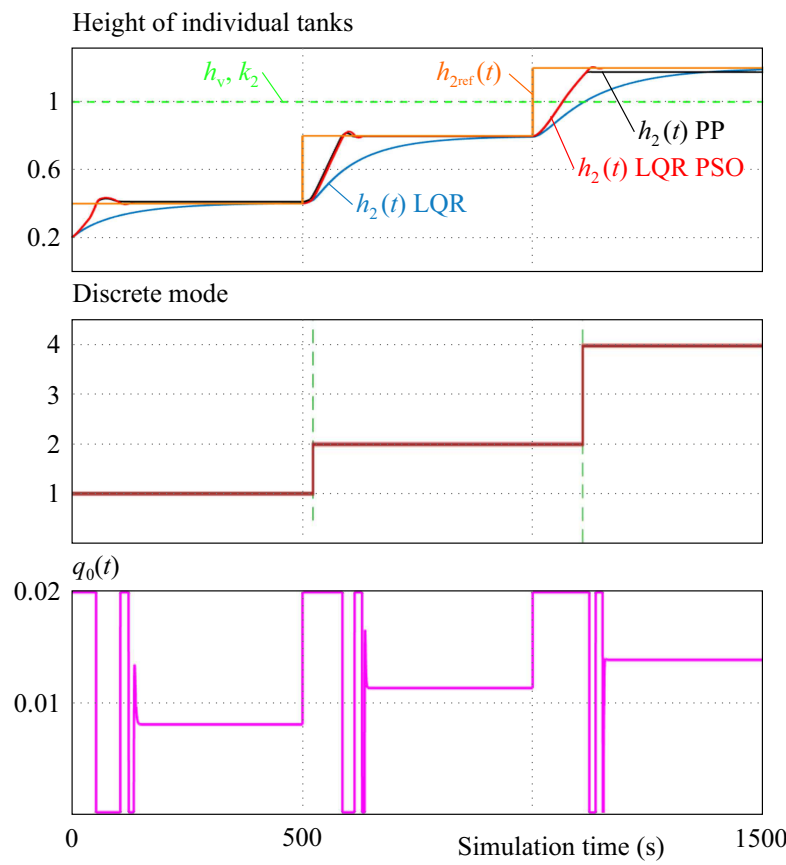

Fig. 11. Set point tracking optimal control of hydraulic HS

The selected weight matrices $\boldsymbol{Q}$ and $\boldsymbol{R}$ for each discrete mode with defined Jacobi matrices were computed based on the PSO algorithm with cost function defined in (14). For comparison random weight matrices $\boldsymbol{Q}, \boldsymbol{R}$ were chosen as $\boldsymbol{Q}_{n}=(100 ; 010)$ and $\boldsymbol{R}_{n}=1$ where $n \in\{\mathcal{A}, \mathcal{B}, \mathcal{D}, \mathcal{F}, \mathcal{H}\}$. Furthermore standard control algorithm based on pole placement method with desired poles -3 and -5 was also added for comparison. Utilizing the PWLQR control algorithm, the height of the level in the second tank $h_{2}(t)$ was able to reach the steady state $h_{2 \text { ref }}(t)$ with any valve $k_{1}$ logical state as seen in Fig. 11.

Verification of the proposed control algorithm was made with regards to set point tracking as well as disturbance rejection. The aim of the control algorithm was in the first case to achieve a transition from discrete mode $\mathcal{A}$ to mode $\mathcal{D}$ via mode $\mathcal{B}$ as seen in Fig. 11 . Figure 12 depicts results for disturbance rejection. Green dashed lines represent transitions between discrete modes.

It can be seen that in both cases PWLQR based on PSO algorithm achieved the best performance either in set point tracking or in disturbance rejection control objective. These results conclude the steps of the proposed methodology.

\section{Conclusions}

This paper introduced one of the main CPS challenges, namely representation of CPS as hybrid dynamical systems. A solution in the form of methodology was proposed for a class of dynamical systems which show hybrid behavior, ie evaluate their state space vector in continuous as well as in discrete state. The methodology comprises several main modules such as Modelling, Analysis and
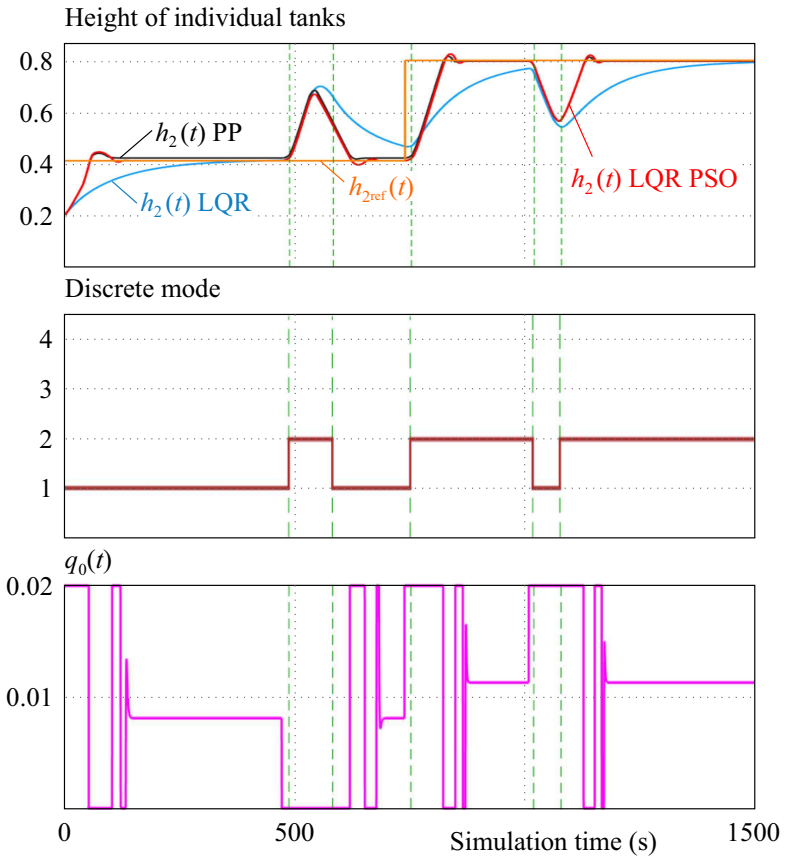

Fig. 12. Piecewise optimal control of hydraulic HS with disturbance rejection

Control design which contain specific steps for aforementioned tasks.

After introduction of the methodology for hybrid systems, a case study of a nonlinear hydraulic hybrid system was provided. The system was described within 8 discrete modes, transitions between some of them are natural with the change of a state space vector, moreover a discrete input action can be applied upon the system. For some of these discrete modes a linear approximation cannot be created in a way using Jacobi matrices. Workaround using straight line instead of square root function was utilized.

Upon completion of the Modelling module, implementation within simulation environment MATLAB/Simulink was performed. With the use of steps from Analysis module, ie phase portrait and open-loop analysis, we conducted a validation of the linear representation of the nonlinear hybrid system.

Having validated hybrid system, it was possible to design control algorithms. Within this paper, piecewise linear-quadratic optimal control tuned by particle swarm optimization algorithm was designed based on the linear approximation of the system and applied onto its nonlinear form.

Future development of the methodology is in the automation of the whole process using the suitable programming environment.

\section{Acknowledgement}

This work has been supported by grant KEGA Implementation of research results in the area of modeling and simulation of cyber-physical systems into the teaching process - development of modern university textbooks - 072TUKE-4/2018. 


\section{REFERENCES}

[1] J. Wan, H. Yan, H. Suo, and F. Li, "Advances in cyber-physical systems research", TIIS, vol. 5, no. 11, pp. 1891-1908, 2011.

[2] D. Vošček and A. Jadlovská, "Modelling and control of a cyber-physical system represented by hydraulic coupled tanks", 15th International Symposium on Applied Machine Intelligence Informatics SAMI, IEEE, 2017.

[3] A. Jadlovská, S. Jadlovská, and D. Vošček, "Cyber-physical system implementation into the distributed control system", IFAC-Papers OnLine, vol. 49, no. 25, pp. 31-36, 2016.

[4] D. Vošček, "Contribution to hybrid models of cyber-physical systems and their implementation into distributed control system", SCYR, pp. 62-63, TU, 2017.

[5] D. Vošček, "Implementation enhancement of hybrid systems modelling within distributed control system", SCYR, pp. 126 -127 , TU, 2018.

[6] Y. Yang and X. Zhou, "Cyber-physical systems modeling based on extended hybrid automata", Computational and Information Sciences (ICCIS), Fifth International Conference, pp. 1871 -1874 , IEEE, 2013.

[7] P. Collins and J. H. Van Schuppen, "Observability of piecewise-affine hybrid systems", International Workshop on $\mathrm{Hy}$ brid Systems: Computation and Control, pp. 265-279, Springer, 2004.

[8] C. Vlad, P. Rodriguez-Ayerbe, E. Godoy, and P. Lefranc, "Advanced control laws of DC-DC converters based on piecewise affine modelling: Application to a step-down converter", IET Power Electronics vol. 7, no. 6, pp. 1482-1498, 2014.

[9] A. Karimoddini, H. Lin, B. M. Chen, and T. H. Lee, "Hierarchical hybrid modelling and control of an unmanned helicopter", International Journal of Control vol. 87, no. 9, pp. 1779-1793, 2014.

[10] E. Chanthery and P. Ribot, "An integrated framework for diagnosis and prognosis of hybrid systems", ArXiv preprint: 1308.5332, 2013.

[11] D. Vošček, A. Jadlovská, and D. Griglak, "Modelling and analysis of the lift system as a hybrid system", Acta Electrotechnica et Informatica vol. 17, no, 4, pp, 28-34, 2017.

[12] M. Herceg, M. Kvasnica, C. N. Jones, and M. Morari, "Multiparametric toolbox 3.0", Control Conference (ECC) 2013 European, pp. 502-510, IEEE, 2013.

[13] M. Johansson and A. Rantzer, "Piecewise linear quadratic optimal control", IEEE Transactions on Automatic Control vol. 45, no, 4, pp, 629-637, 2000.

[14] L. Zhang and G. Li, "Controller design for discrete-time hybrid linear parameter-varying systems with semi-Markov mode switching", Journal of the Franklin Institute vol. 355, no. 15, pp. 7056-7071, 2018.

[15] X. D. Koutsoukos, P. J. Antsaklis, J. A. Stiver, and M. D. Lemmon, "Supervisory control of hybrid systems", Proceedings of the IEEE vol. 88, no. 7, pp. 1026-1049, 2000.

[16] E. V. Kumar, G. S. Raaja, and J. Jerome, "Adaptive PSO for optimal LQR tracking control of 2 DOF laboratory helicopter", Applied Soft Computing vol. 41, pp. 77-90, 2016.

[17] H. Wang, H. Zhou, D. Wang, and S. Wen, "Optimization of LQR controller for inverted pendulum sysem with artificial bee colony algorithm", in Proceedings of the 2013 International Conference on Advanced Mechatronic Systems, pp. 158-162, IEEE, 2013.

[18] A. Jacknoon and M. Abido, "Ant colony based LQR PID tuned parameters for controlling inverted pendulum", in 2017 International Conference on Communication Control, Computing Electronics Engineering (ICCCCEE), pp. 1-8, IEEE, 2017.

[19] N. Razmjooy, M. Ramezani, and A. Namadchian, "A new LQR optimal control for a single-link flexible joint robot manipulator based on grey wolf optimizer", Majlesi Journal of Electrical Engineering vol. 10, no. 3, pp. 53, 2016.
[20] D. Vošček, "Contribution to hybrid models of cyber-physical systems their implementation into distributed control system", Thesis TU, 2017, Supervisor: Anna Jadlovská.

[21] Q. Wang, G. Yang, X. Zhou, and Y. Yang, "Behavior modeling of cyber-physical system based on discrete hybrid automata", Computational Science Engineering (CSE) 2013 IEEE 16th International Conference, pp. 680-684, IEEE, 2013.

22] J. Lygeros, K. H. Johansson, S. N. Simic, J. Zhang, and S. S. Sastry, "Dynamical properties of hybrid automata", IEEE Transactions on Automatic Control vol. 48, no, 1, pp, 2-17, 2003.

[23] H. Lin and P. J. Antsaklis, "Hybrid dynamical systems: An introduction to control verification", Foundations Trends in Systems Control vol. 1, no, 1, pp, 1-172, 2014.

[24] A. Bemporad, "Hybrid Toolbox - User's Guide", http://cse.lab.imtlucca.it/ ${ }^{\sim}$ bemporad/hybrid/toolbox, 2004

[25] M. H. M. Kvasnica, HYSDEL 3.0, Manual STU.

[26] A. Haraux and M. A. Jendoubi, "The linearization method in stability analysis", The Convergence Problem for Dissipative Autonomous Systems, pp. 45-65, Springer, 2015.

[27] K. Halbaoui, M. Belazreg, D. Boukhetala, and M. Belhouchat, "Modeling and predictive control of nonlinear hybrid systems using mixed logical dynamical formalism", in Advances Applications in Nonlinear Control Systems, pp. 421-450, Springer, 2016.

28] I. R. Manchester, M. M. Tobenkin, M. Levashov, and R. Tedrake, "Regions of attraction for hybrid limit cycles of walking robots", IFAC Proceedings vol. 44, no. 1, pp. 5801-5806, 2011.

[29] F. Zhu and P. J. Antsaklis, "Optimal control of hybrid switched systems: A brief survey", Discrete Event Dynamic Systems vol. 25 , no. 3 , pp. $345-364,2015$

Received 19 March 2019

Dominik Vošček graduated (MSc) with honors at the Department of Cybernetics and Artificial Intelligence of the Faculty of Electrical Engineering and Informatics at Technical University of Košice in 2015. Since September 2015, he has been internal PhD student at the same department focusing on the research of cyber-physical systems utilizing hybrid system framework within thesis: Hybrid Models of CyberPhysical Systems and their Application into Distributed Control Systems.

Anna Jadlovská received her MSc degree in the field of Technical Cybernetics at the Faculty of Electrical Engineering of the Technical University in Košice in 1984. She defended her $\mathrm{PhD}$ thesis in the domain of Automatization and Control in 2001 at the same University; her thesis title was Modelling and Control of Nonlinear Processes Using Neural Networks. Since 1993 she worked in the Department of Cybernetics and Artificial Intelligence Faculty of Electrical Engineering and Informatics Technical University in Košice as an Associate Assistant and since 2004 she has been working as an Associate Professor. Her main research activities include the problems of modelling and optimal control of hybrid nonlinear dynamical systems using effective simulation tools.

Dominik Griglák is a student at the Department of $\mathrm{Cy}$ bernetics and Artificial Intelligence of the Faculty of Electrical Engineering and Informatics at Technical University of Košice. In 2017, he received his bachelor degree after defending his bachelor thesis: Modelling, Analysis, Control and Simulation of Hybrid systems, focused on designing a program tool with the application for modeling of hybrid systems. 